\title{
Clinical Development Plan
}

National Cancer Institute

\section{Source}

National Cancer Institute. Clinical Development Plan. NCI Thesaurus. Code C142424.

A descriptive record of the clinical investigations to be executed with a particular treatment, substance or device. This is usually done to obtain marketing approval. 